Supporting Information for

\title{
Graphene Thermal Emitter with Enhanced Joule Heating and Localized Light Emission in Air
}

Fang Luo ${ }^{1, *}$, Yansong Fan ${ }^{1, *}$, Gang Peng ${ }^{2}$, Shuigang Xu ${ }^{3}$, Yaping Yang ${ }^{3}$, Kai Yuan $^{4}$, Jinxin Liu ${ }^{5}$,

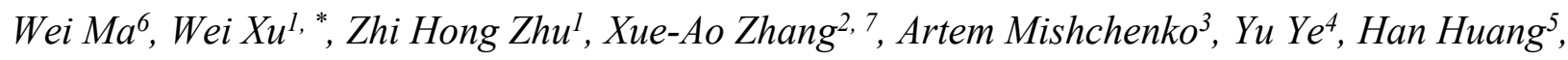
Zheng Han ${ }^{6}$, Wencai Ren ${ }^{6}$, Kostya S. Novoselov ${ }^{3}$, Mengjian Zhu ${ }^{1,}$ *, Shiqiao Qin ${ }^{1}$

Corresponding to: weixu08a@163.com; zhumengjian11@nudt.edu.cn

Section 1. Device fabrication and characterization

Figure S1-Figure S5

Section 2. Raman spectroscopy and lattice temperature measurements

Figure S6-Figure S11

Section 3. Simulation of temperature distribution

Figure S12-Figure S14

Section 4. Simulation of the electromagnetic field distribution in $\mathrm{hBN} / \mathrm{graphene} / \mathrm{hBN}$ thermal emitters

Figure S15

Section 5. Enhanced emission by a photonic cavity

Section 6. Resonant emission at telecom spectrum range

Figure S16-Figure S17 


\section{Section 1. Device fabrication and characterization}

\subsection{Bare graphene devices}

A typical device consists of a monolayer graphene flake on a degenerately doped Si substrate with a $300 \mathrm{~nm} \mathrm{SiO}_{2}$ on top. The monolayer graphene was mechanically exfoliated from graphite (HQ graphene) and then transferred onto the Si substrate. Raman spectroscopy was applied to identify the number of layers of graphene (see in Figure S1). The graphene flakes were etched to rectangular strips with a fixed width $(W)$ of $4 \mu \mathrm{m}$. Several graphene strips were further etched to bowties shape with constrictions at the middle. The width of constrictions is $1.5 \mu \mathrm{m}$ and $1.0 \mu \mathrm{m}$, respectively (see in Figure S2). The constrictions were prepared by patterning PMMA resists with electron-beam lithography. The uncovered graphene was then etched away by $\mathrm{CHF}_{3}+\mathrm{O}_{2}$ plasma to form the bowties geometry. Graphene stripes were contacted by $\mathrm{Cr} / \mathrm{Au}(5 \mathrm{~nm} / 50 \mathrm{~nm})$ metal leads using electron-beam evaporation. The distance between the two contacts (device length, $L$ ) is fixed at $10 \mu \mathrm{m}$. 

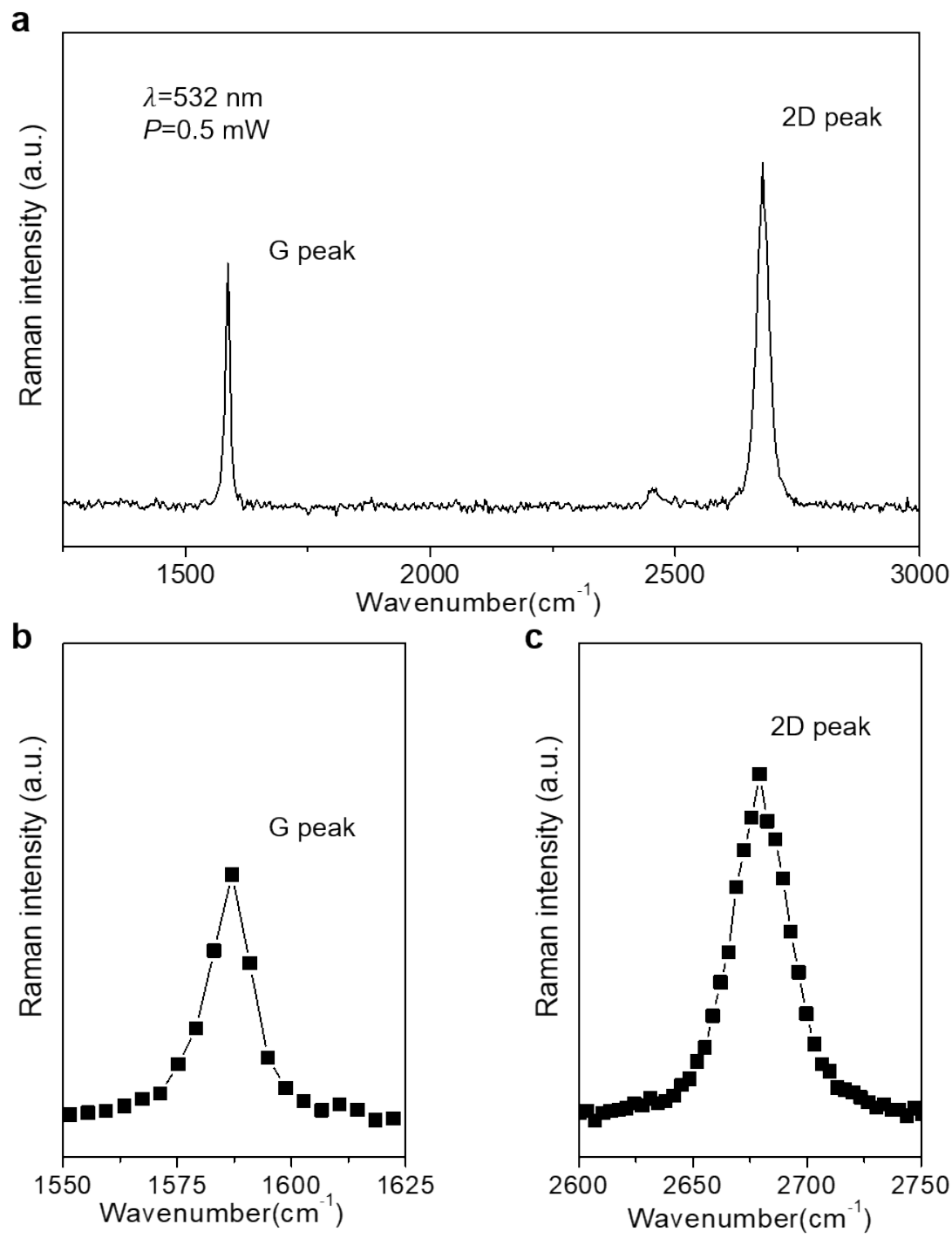

Figure S1. Raman spectroscopy of a monolayer graphene. a Measured Raman spectroscopy of a monolayer graphene (Gr device in the maintext) with $\mathbf{b}$ G peak at $\sim 1590 \mathrm{~cm}^{-1}$ and $\mathbf{c} 2 \mathrm{D}$ peak at $\sim 2680 \mathrm{~cm}^{-1}$. 

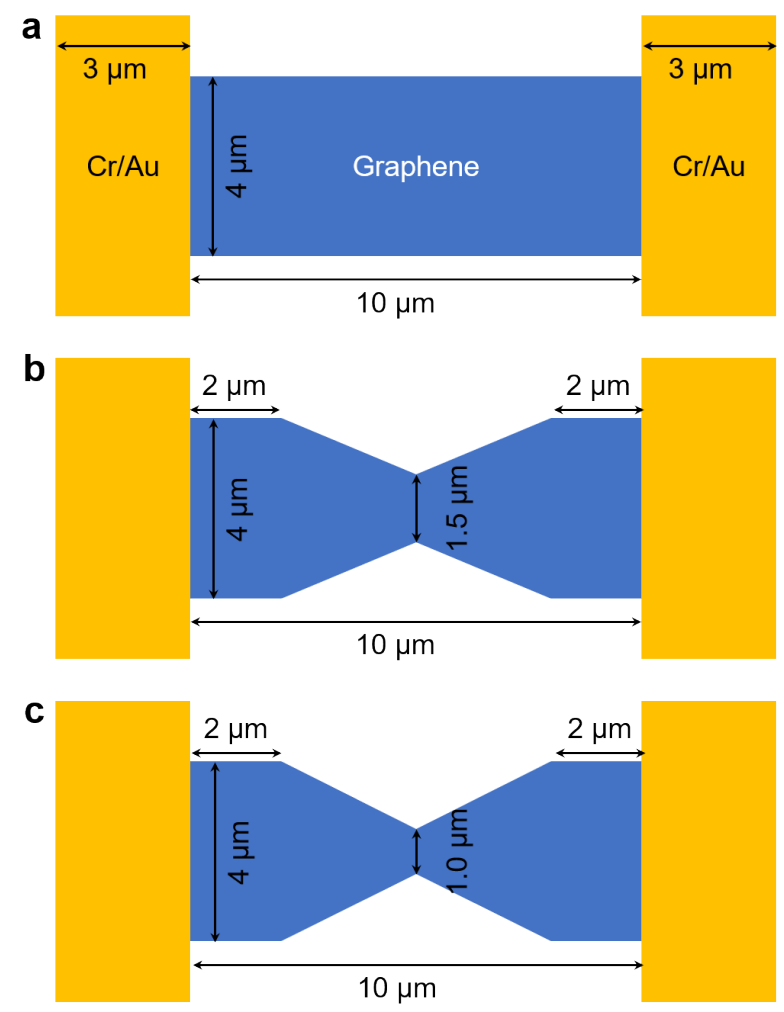

Figure S2. The geometry of graphene devices. a Rectangular graphene, Gr. b, c Bowtie graphene constriction Gr-C-1.5 $\mu \mathrm{m}$ and $\mathrm{Gr}-\mathrm{C}-1.0 \mu \mathrm{m}$, respectively.
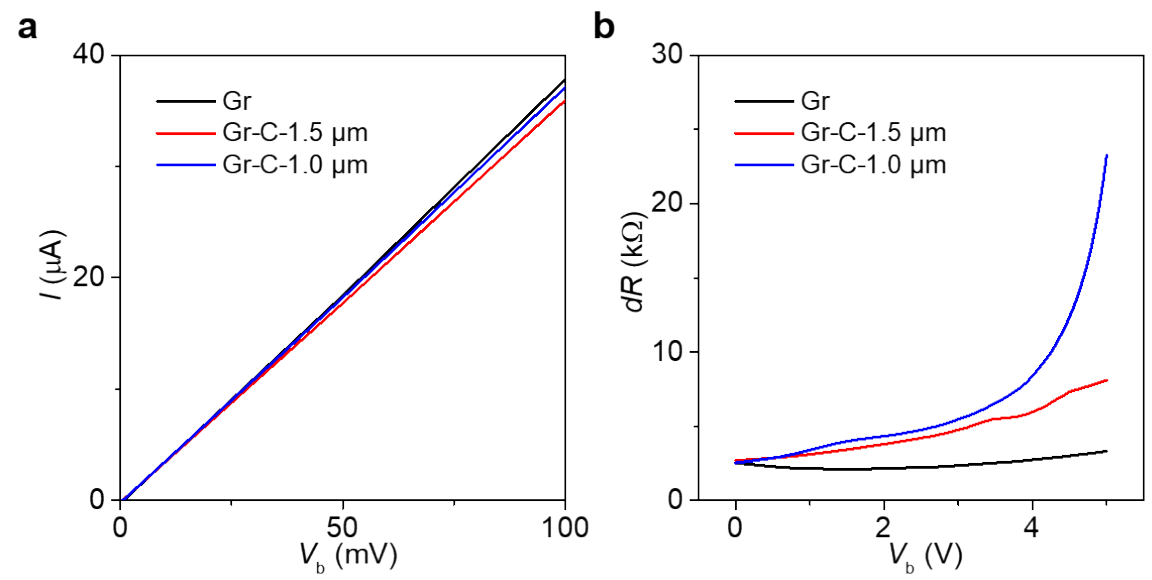

Figure S3. Electric transport properties of graphene devices. a Measured $I-V_{\mathrm{b}}$ curves and b calculated differential resistance $(\mathrm{d} R=\mathrm{d} V / \mathrm{d} l)$ for differnt graphene devices. 


\section{2 hBN encapsulated graphene devices}

To demonstrate the visible light emission, we fabricated hexagonal boron nitride (hBN) encapsulated graphene constrictions. Both hBN (HQ graphene) flakes and monolayer graphene are prepared by mechanical exfoliation. The $\mathrm{hBN} / \mathrm{Gr} / \mathrm{hBN}$ van der Waals heterostructures were assembled using a dry pick-up method and transferred onto $\mathrm{SiO}_{2} / \mathrm{Si}$ substrates with a $300 \mathrm{~nm} \mathrm{SiO}_{2}$ on top. The thickness of heterostructure is $\sim 50 \mathrm{~nm}$ in total measured by atomic force miscroscopy (see in Figure S4).The heterostructures were etched to form the constriction and contact profiles. The one-dimensional (1D) contacts were formed by depositing $\mathrm{Cr} / \mathrm{Au}(5 \mathrm{~nm} / 50 \mathrm{~nm})$ leads along the two exposed graphene edges. Such device architecture allows a very clean platform for the investigation of Joule heating, energy dissipation and thermal emission in graphene.
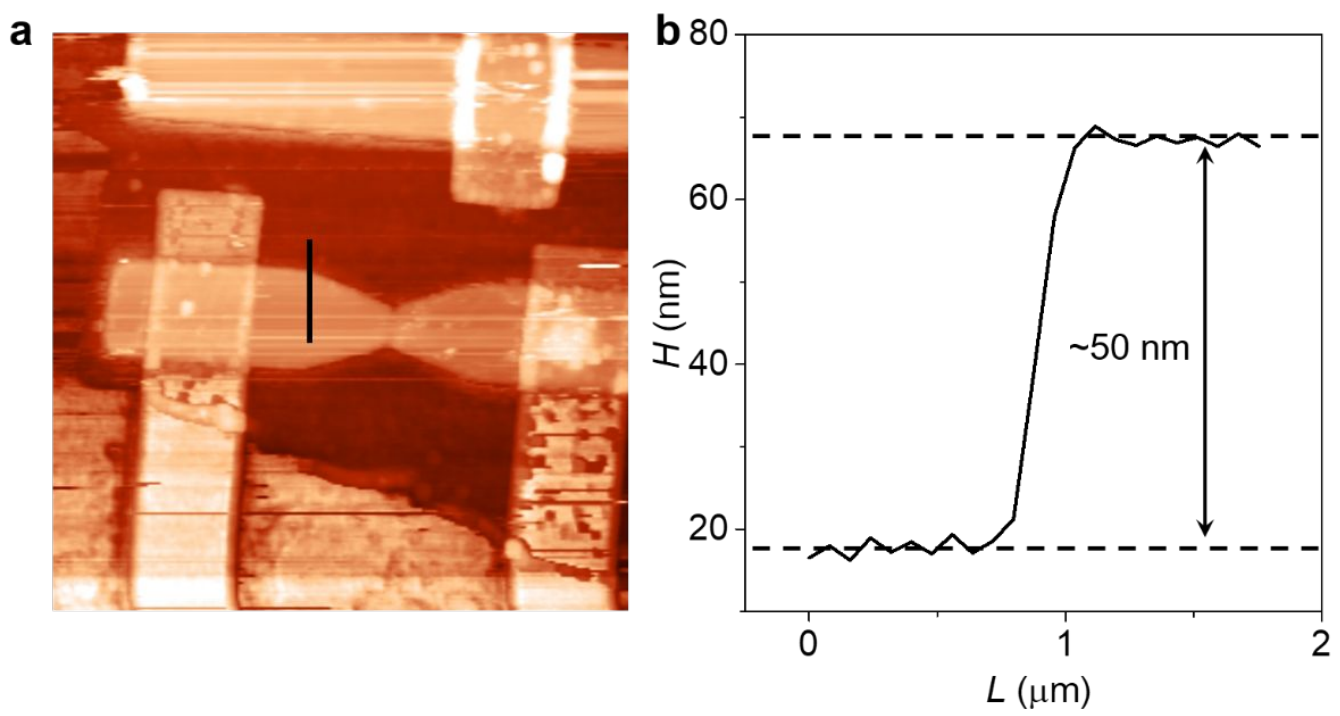

Figure S4. AFM measurement of the thickness of $\mathrm{hBN} / \mathrm{Gr} / \mathrm{hBN}$ heterostructures. a AFM mapping of device. $\mathbf{b}$ The height profile along the black line in Figure S4a. 


\section{$1.3 \mathrm{Al}_{2} \mathrm{O}_{3}$ capped graphene devices}

We fabricated an $\mathrm{Al}_{2} \mathrm{O}_{3}$ capped graphene emitter with a $1.5 \mu \mathrm{m}$ constriction at the middle $\left(\mathrm{Al}_{2} \mathrm{O}_{3^{-}}\right.$ Gr-C-1.5 $\mu \mathrm{m}$ ) by depositing a $2 \mathrm{~nm}$ thick Al film on graphene and followed by an ALD growth of a $70 \mathrm{~nm}$ thick $\mathrm{Al}_{2} \mathrm{O}_{3}$ film. Here we also used the monolayer graphene from mechanical exfoliation. After the fabrication of a bare graphene constriction device (Gr-C-1.5 $\mu \mathrm{m})$, we deposited a $2 \mathrm{~nm}$ thick Al thin film on top of the device using magnet controlled sputtering method. After that, the device was exposed in air for half an hour in order to form the $\mathrm{Al}_{2} \mathrm{O}_{3}$ layer due to oxidation of $\mathrm{Al}$. Finally, we grew a $70 \mathrm{~nm}$ thick $\mathrm{Al}_{2} \mathrm{O}_{3}$ film on top of the graphene device by atomic layer deposition (ALD) method.

\section{$1.4 \mathrm{Al}_{2} \mathrm{O}_{3}$ capped graphene emitters array}

Monolayer graphene with centimeter-size single crystal was grown by CVD method and then was transferred onto $\mathrm{SiO}_{2} / \mathrm{Si}$ substrate by electro-chemical bubbling method (see in Figure S5). After patterning a $4 \times 4$ graphene array and depositing of electrodes, we grew a $70 \mathrm{~nm}$ thick $\mathrm{Al}_{2} \mathrm{O}_{3}$ capping layer by magnet controlled sputtering and atomic-layer-deposition (ALD) method.
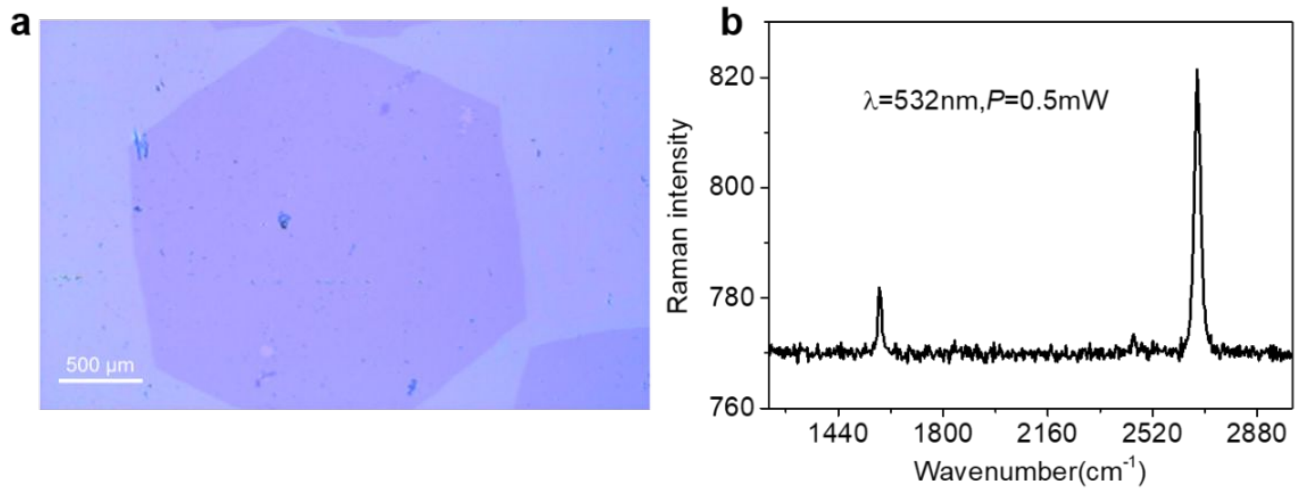

Figure S5. Monolayer graphene grown by CVD method. a Optical micrograph of CVD grown graphene after transferred onto $\mathrm{SiO}_{2} / \mathrm{Si}$ substrate. b Raman spectrum of CVD graphene. 


\section{Section 2. Raman spectroscopy and lattice temperature measurements}
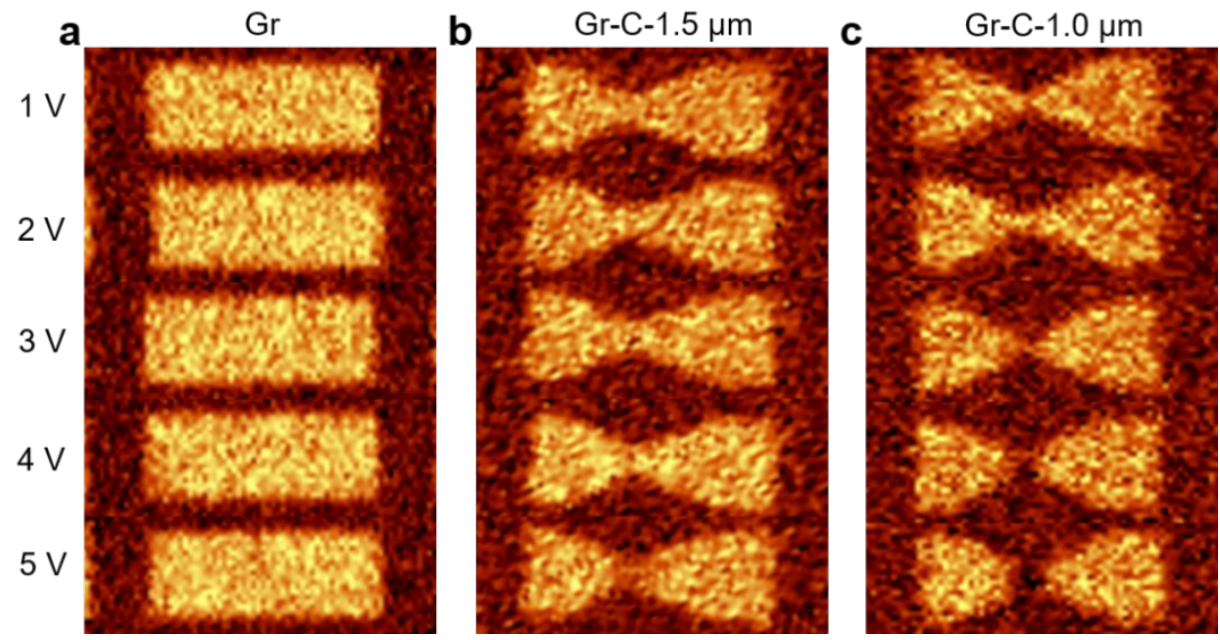

Figure S6. Raman maps of graphene devices at various bias voltages. a Graphene strip without constriction, Gr. b Graphene bowtie with $1.5 \mu \mathrm{m}$ width constriction, Gr-C-1.5 $\mu \mathrm{m}$. c Graphene bowtie with $1.0 \mu \mathrm{m}$ width constriction, Gr-C-1.0 $\mu \mathrm{m}$.
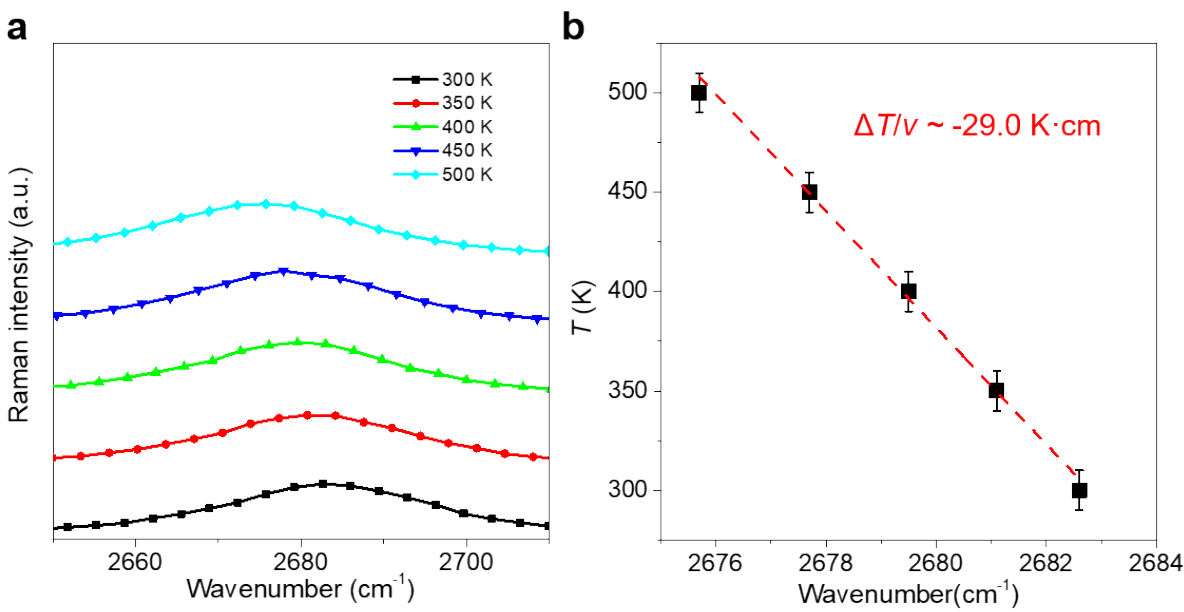

Figure S7. Calibration of the ratio between Raman shift of graphene 2D peaks and the lattice temperature. a Measured Raman 2D bands of graphene as a function of the temperature of a local heated stage with graphene on top. b Plotted 2D peak positions against the temperature. The dash line is the linear fit, showing $\Delta T / v \sim-29.0 \mathrm{~K} \cdot \mathrm{cm}$. 

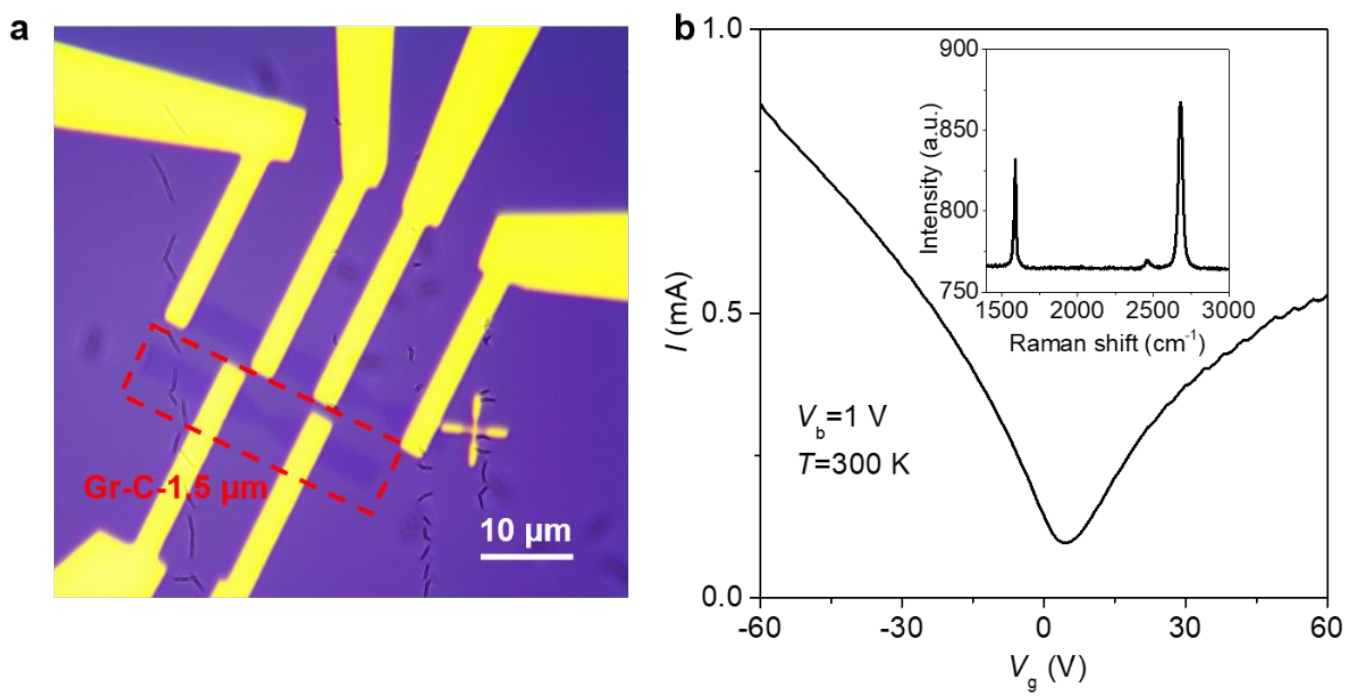

Figure S8. Characterizations of a back-gated graphene device, Gr-C-1.5 $\mu \mathrm{m}$. a Optical microscopic image of graphene devices. Gr-C-1.5 $\mu \mathrm{m}$ is highlighted by the red dashed line. b Measured current $I$ as a function of $V_{\mathrm{g}}$. The inset shows the Raman spectroscopy of the monolayer graphene with G mode at $1590 \mathrm{~cm}^{-1}$ and 2D mode at $2681 \mathrm{~cm}^{-1}$.

Table S1. 2D peak positions of graphene under various bias and gate voltages

\begin{tabular}{|l|l|l|l|}
\hline$V_{\mathrm{g}}, V_{\mathrm{b}}$ & $0 \mathrm{~V}$ & $4 \mathrm{~V}$ & $8 \mathrm{~V}$ \\
\hline $0 \mathrm{~V}$ & 2681.1 & 2673.1 & 2661.8 \\
\hline $40 \mathrm{~V}$ & 2681.4 & 2673.7 & 2664.3 \\
\hline$-40 \mathrm{~V}$ & 2681.6 & 2673.3 & 2662.4 \\
\hline
\end{tabular}



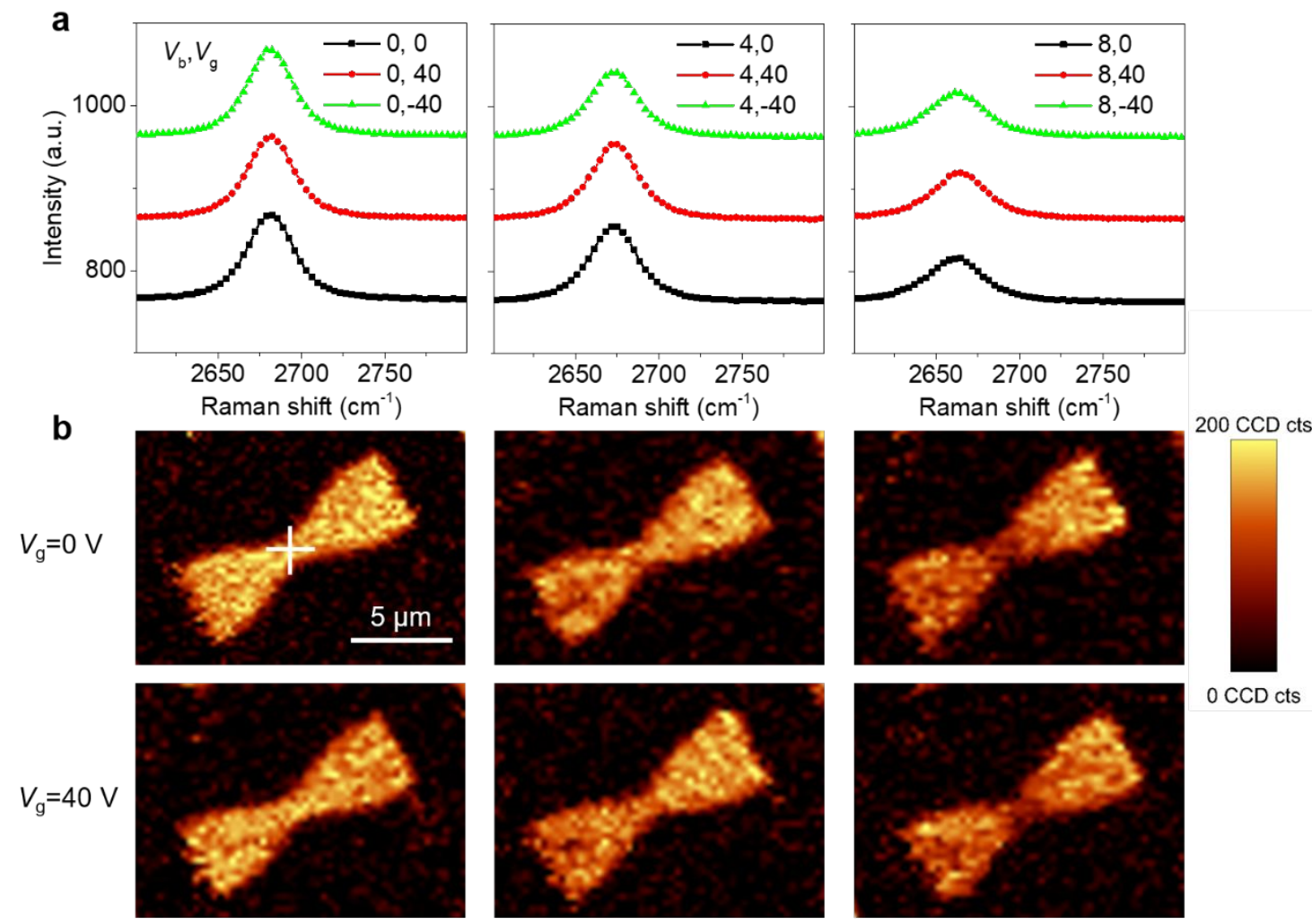

$0 \mathrm{CCD}$ cts
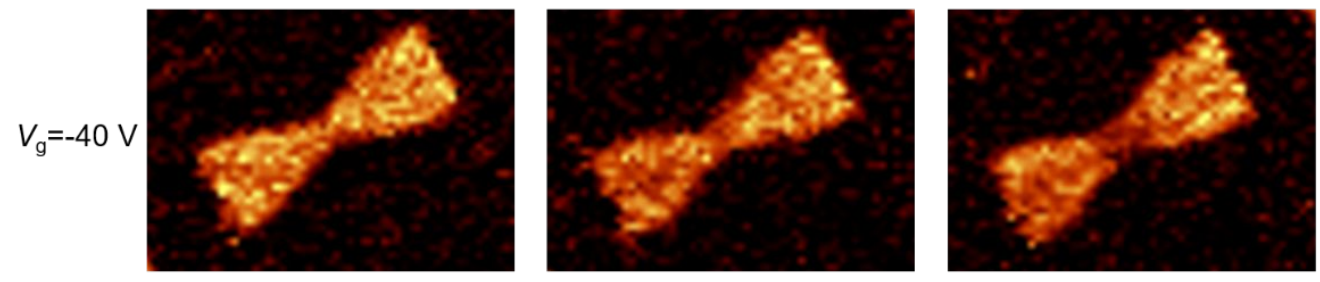

$V_{\mathrm{b}}=0 \mathrm{~V}$

$V_{\mathrm{b}}=4 \mathrm{~V}$

$$
V_{\mathrm{b}}=8 \mathrm{~V}
$$

Figure S9. Measured Raman 2D modes of graphene under various $V_{\mathrm{g}}$ and $V_{\mathrm{b}}$. a Raman 2D bands of graphene for $V_{\mathrm{b}}=0 \mathrm{~V}, 4 \mathrm{~V}, 8 \mathrm{~V}$ and $V_{\mathrm{g}}=0 \mathrm{~V}, 40 \mathrm{~V},-40 \mathrm{~V}$, respectively. The Raman measurements are taken at the middle of constriction, marked by the white cross in Figure S9b. b Raman maps of graphene constriction under various $V_{\mathrm{g}}$ and $V_{\mathrm{b}}$. The maps are defined by the 2D peak with a central wavenumber $2680 \mathrm{~cm}^{-1}$ and a width $80 \mathrm{~cm}^{-1}$. 

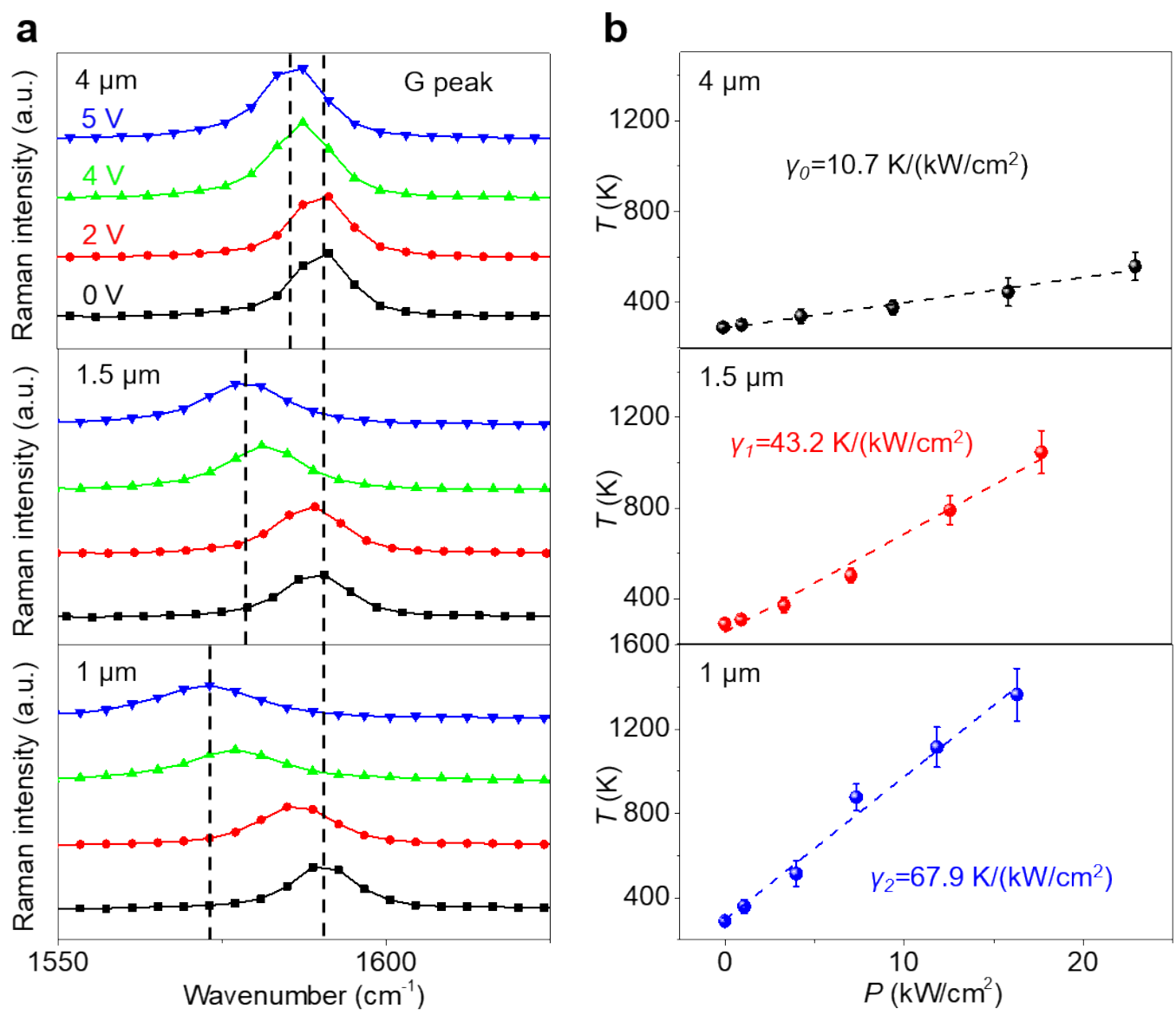

Figure S10. G peak derived lattice temperature of biased graphene devices. a Raman spectrum of graphene under various $V_{\mathrm{b}}$ from $0 \mathrm{~V}$ to $5 \mathrm{~V}$. b Measured temperature $T$ of graphene at the central as a function of electrical power density $p$. 

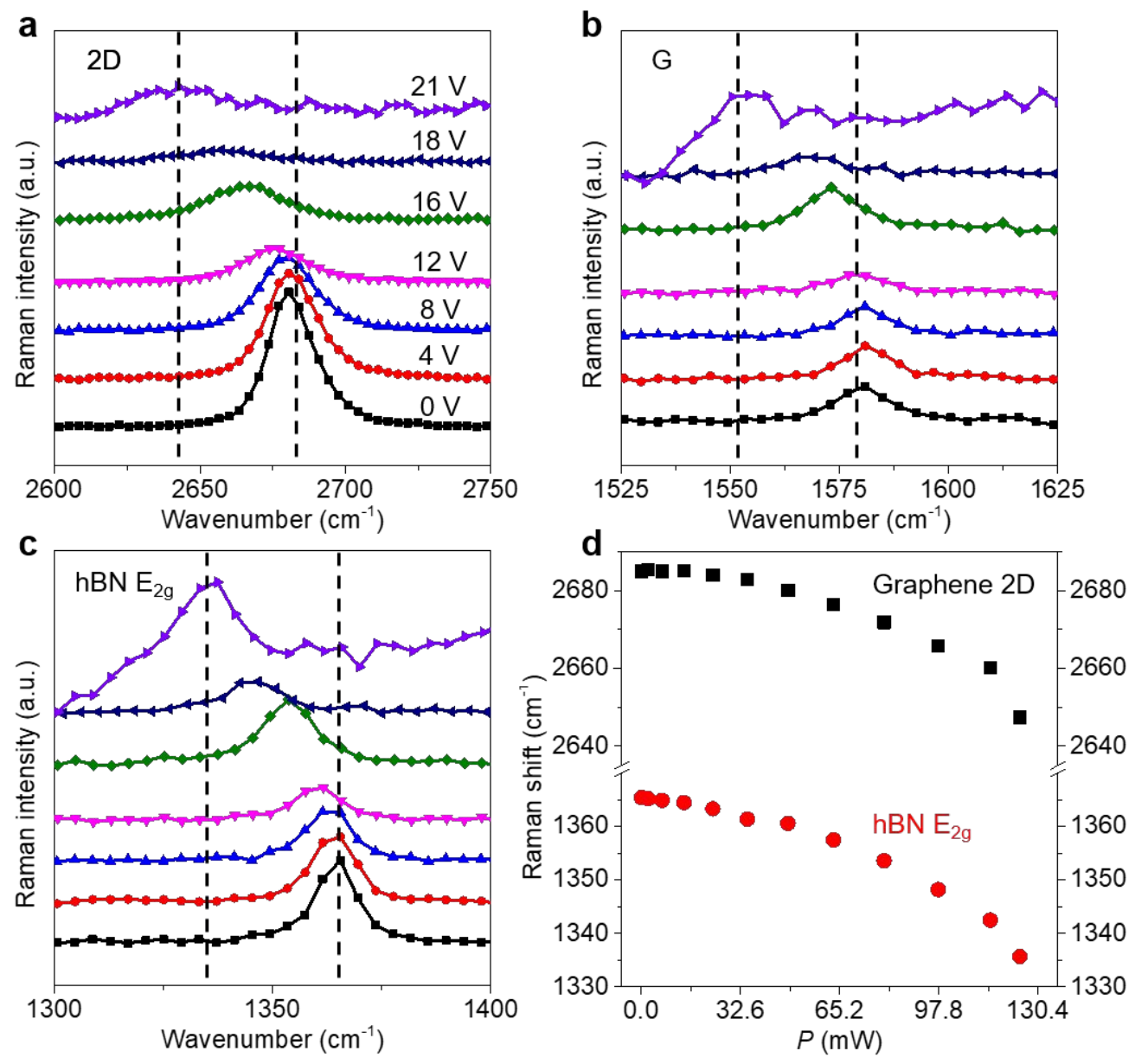

Figure S11. Raman spectra of $\mathrm{hBN} / \mathrm{Gr} / \mathrm{hBN}$ heterostructure under different bias voltage. a, b Raman spectra of 2D peak and $\mathrm{G}$ peak of graphene, respectively. $\mathbf{c}$ Raman spectra of $\mathrm{E}_{2 \mathrm{~g}}$ peak of $\mathrm{hBN}$. d The downshift of graphene $2 \mathrm{D}$ peak and $\mathrm{hBN} \mathrm{E}_{2 \mathrm{~g}}$ peak as a function of supplied electrical power density. 


\section{Section 3. Simulation of temperature distribution}

We first estimated the thermal radiation efficiency (the ratio of thermal radiation power over electrical power input) of our devices, we calculated the total thermal radiation power given by the Stefan-Boltzmann law, $J=\epsilon \sigma T^{4}$, where $\epsilon=0.023$ for monolayer graphene, $\sigma=5.67 \times 10^{-8} \mathrm{~W} /$ $\mathrm{m}^{2} \mathrm{~K}^{4}$ is Stefan's constant and $\mathrm{T}$ is the electron temperature derived from the light emission spectra. For the case of $\mathrm{hBN} / \mathrm{Gr} / \mathrm{hBN}-\mathrm{C}-1.5 \mu \mathrm{m}$ device, the electron temperature is about $2100 \mathrm{~K}$ at applied electrical power of $100 \mathrm{KW} / \mathrm{cm}^{2}$. The radiation power by the Stefan-Boltzman law is $2.54 \mathrm{~W} / \mathrm{cm}^{2}$ , so energy dissipation through radiation is about $2.54 \times 10^{-5}$. The radiation efficiency of other devices in the main text are less than or on the same order of that of the $\mathrm{hBN} / \mathrm{Gr} / \mathrm{hBN}-\mathrm{C}-1.5 \mu \mathrm{m}$ device. So We ignore the thermal radiation power and only solve the heat diffusion equation $-\nabla$. $(\kappa \nabla T)=\dot{q}$ by three-dimensional finite element method to investigte the temperature distribution in electrically biased graphene. In our simulation, the thermal conductivity of $\mathrm{SiO}_{2}, \mathrm{Si}, \mathrm{Al}_{2} \mathrm{O}_{3}$, and metal contacts are $1.4 \mathrm{~W} /(\mathrm{m} \cdot \mathrm{K}), 50 \mathrm{~W} /(\mathrm{m} \cdot \mathrm{K}), 30 \mathrm{~W} /(\mathrm{m} \cdot \mathrm{K})$ and $317 \mathrm{~W} /(\mathrm{m} \cdot \mathrm{K})$, respectively. The in-plane and out of plane thermal conductivity of $\mathrm{hBN}$ are set as $2.5 \mathrm{~W} /(\mathrm{m} \cdot \mathrm{K})$ and $250 \mathrm{~W} /(\mathrm{m} \cdot \mathrm{K})$, respectively. The thermal conductivity of graphene is determined by the temperature of acoustic phonon $\kappa\left(T_{a p}\right)=\kappa\left(T_{0}\right)\left(T_{0} / T_{a p}\right)^{\gamma}$. For the case of graphene supported by $\mathrm{SiO}_{2}$, we used $\kappa\left(T_{0}\right)=550 \mathrm{~W} /(\mathrm{m} \cdot \mathrm{K}), \gamma=0.8$. While, for the case of graphene encapsulated by hBN, we used $\kappa\left(T_{0}\right)=2000 \mathrm{~W} /(\mathrm{m} \cdot \mathrm{K})$, The interface between graphene and the underlying $\mathrm{SiO}_{2}$ $(\mathrm{hBN})$ is modeled with thermal interface resistance of $4.2 \times 10^{-7} \mathrm{~m}^{2} \mathrm{~K} / \mathrm{W}\left(2 \times 10^{-8} \mathrm{~m}^{2} \mathrm{~K} / \mathrm{W}\right)$, while the interface between metal contacts and graphene is modeled as intimate contact (thermal resistance set to zero). 
The input power flux $\dot{q}=\mathcal{J} \mathrm{E}$, where $\mathrm{E}=\nabla \mathrm{V}$ is the local electric field and $\mathrm{V}$ is the electric potential in graphene due to the applied source-drain bias voltage. The electric current $\mathcal{J}=\sigma E$, where the conductivity of graphene follows $\sigma=\left(n_{e}+n_{h}\right) e \mu\left(n, T_{e}\right)$. The electron (hole) carrier density is $n_{e}$ $\left(n_{h}\right)=\frac{1}{2}\left( \pm n_{d}+\sqrt{n_{d}^{2}+4 n_{t h}^{2}+n_{p d}^{2}}\right)$, where the thermally excited carrier density of graphene is $n_{t h}=\frac{\pi}{6}\left(\frac{k_{B} T_{e}}{\hbar v_{F}}\right)^{2}\left(1+e^{-\left(\frac{T_{e}}{T_{0}}-1\right) / 2} \sqrt{\left.\frac{T_{e}}{T_{0}}-1\right)}\right.$, the substrate doping carrier density is $n_{d}=C_{o x} V_{D r a c} / e$ and $n_{p d}$ is the carrier density due to electron-hole puddles in graphene. $C_{o x}$ is the gate capacitance, $V_{D r a c}$ is a charge neutral point, $T_{0}$ is the ambient temperature, $e$ and $v_{F}$ are the electron charge and the Fermi velocity of graphene, respectively. The temperature of elctrons and acoustic phonon follows $T_{e}=T_{a p}+\eta\left(T_{a p}-T_{0}\right)$.

For the case of graphene supprted by $\mathrm{SiO}_{2}$, the carrier mobility of graphene dependent on the carrier density and temperature, and is given by

$$
\mu\left(n, T_{e}\right)=\frac{\mu_{0}}{1+\left(n / n_{r e f}\right)^{\alpha}} \times \frac{1}{1+\left(T_{e} / T_{0}-1\right)^{\beta}}
$$

In our model, we have $V_{\text {Drac }}=17 \mathrm{~V}, n_{p d}=2.63 \times 10^{11} \mathrm{~cm}^{-2}, \mu_{0}=5500 \mathrm{~cm}^{2} /(\mathrm{V} \cdot s)$ is the carrier mobility of graphene at $300 \mathrm{~K}, n_{\text {ref }}=1.1 \times 10^{13} \mathrm{~cm}^{-2}, T_{0}=300 \mathrm{~K}, \alpha=2.2, \beta=2.3$ and $\eta=0.7$. The simulation results are shown in maintext Figure $2 \mathrm{~d}-\mathrm{f}$, which agree well with the experimental results. For the case of graphene supported by $\mathrm{SiO}_{2}$ and covered by $\mathrm{Al}_{2} \mathrm{O}_{3}, V_{\text {Drac }}$ $=11 \mathrm{~V}, \mu_{0}=5000 \mathrm{~cm}^{2} /(\mathrm{V} \cdot \mathrm{s})$, with other parameters the same as the case of graphene suppoerted by $\mathrm{SiO}_{2}$. For the case of graphene encapsulated by hBN, $V_{\operatorname{Drac}}=7 \mathrm{~V}, \mu\left(T_{e}\right)=\mu_{0}$ $\left(T_{0} / T_{e}\right)^{\beta}, \mu_{0}=30000 \mathrm{~cm}^{2} /(\mathrm{V} \cdot s), \beta=2.3$. 
In our simulation, convective heat flux boundary condition is used in our model to describe the heat transfer between air and the up boundary of our devices. The heat transfer coefficient is set as $5 \mathrm{~W} / \mathrm{m}^{2} \mathrm{~K}$. Fixed temperature $(T=300 \mathrm{~K})$ boundary condition is used at the boundary of the substrate. The simulated temperature distribution of graphene encapsulated by $\mathrm{hBN}$ and $\mathrm{Al}_{2} \mathrm{O}_{3}$ is shown in Figure S12 and S13, respectively.

\section{1 hBN encapsulated graphene devices}
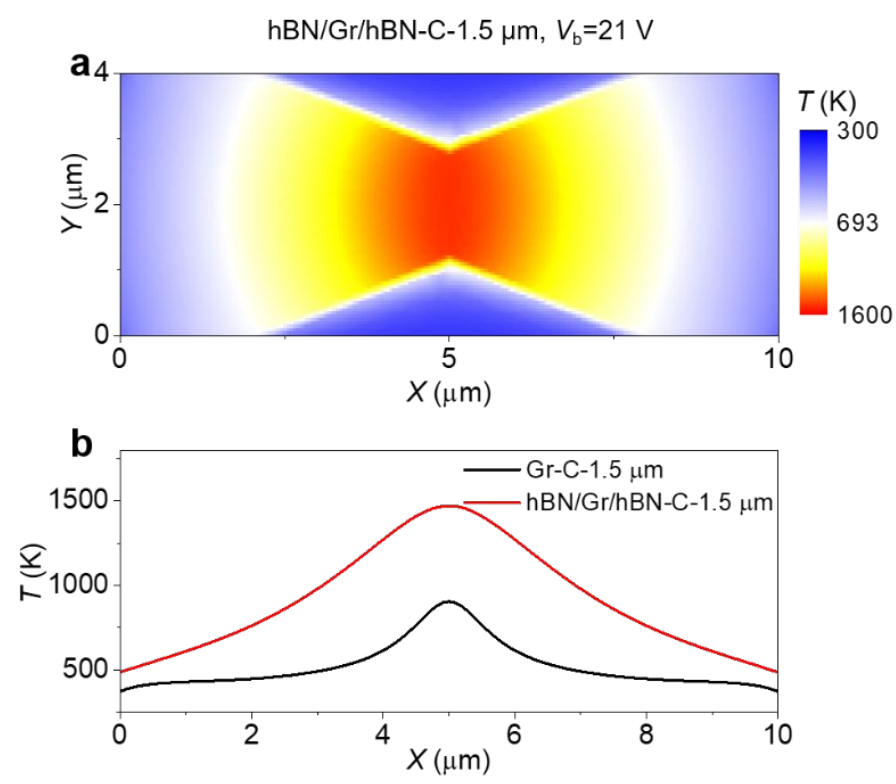

Figure S12. Calculated temperature distribution of hBN encapsulated graphene device $\mathrm{hBN} / \mathrm{Gr} / \mathrm{hBN}-\mathrm{C}-1.5 \mu \mathrm{m}$. a Spatially resolved temperature map. b Temperature along the $x$ axis of $\mathrm{hBN}$ encapsulated graphene and bare graphene constrictions, respectively. 


\section{2 $\mathrm{Al}_{2} \mathrm{O}_{3}$-capped graphene devices}

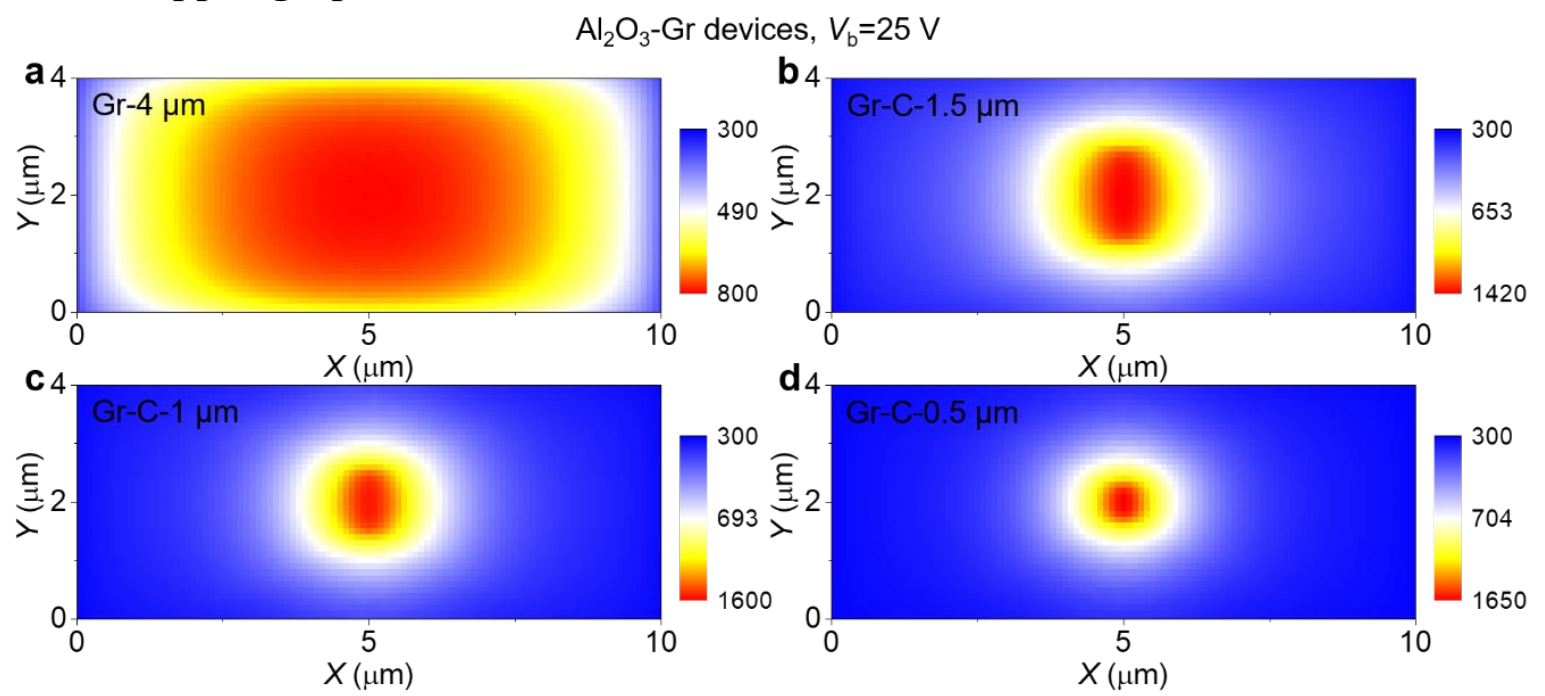

Figure S13. Calculated temperature distribution maps for $\mathrm{Al}_{2} \mathrm{O}_{3}$-capped graphene emitters

\subsection{Vertical temperature distribution in graphene devices}
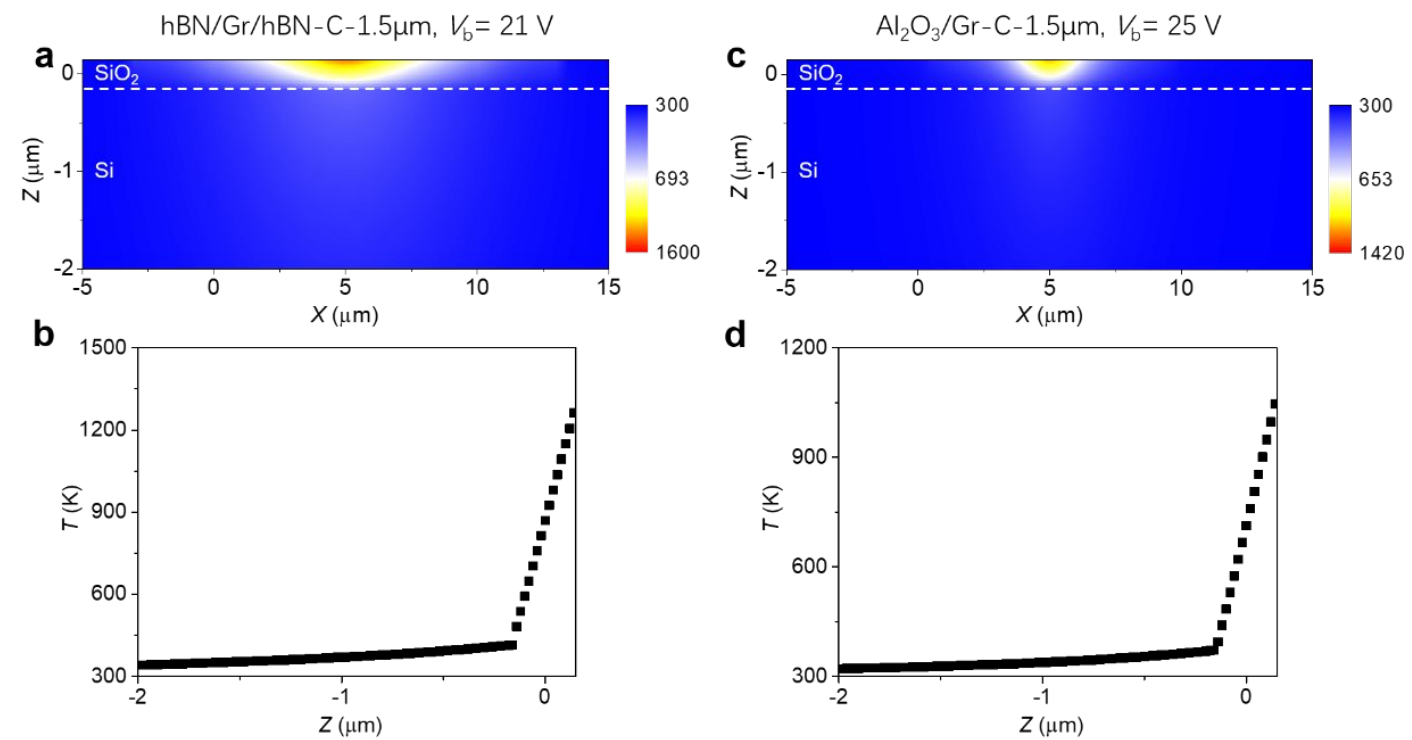

Figure S14. Calculated vertical temperature distribution inside the $\mathrm{SiO}_{2}$ layer and $\mathrm{Si}$ substrate. a,

$\mathbf{b}$ and $\mathbf{c}, \mathbf{d}$ are spatially resolved temperature maps and temperature profiles along the $\mathrm{z}$ axis of $\mathrm{hBN} / \mathrm{Gr} / \mathrm{hBN}-\mathrm{C}-1.5 \mu \mathrm{m}$ device and $\mathrm{Al}_{2} \mathrm{O}_{3} / \mathrm{Gr}-\mathrm{C}-1.5 \mu \mathrm{m}$ device, respectively. 


\section{Section 4. Simulation of the electromagnetic field distribution in $\mathrm{hBN} / \mathrm{graphene} / \mathrm{hBN}$ thermal}

emitters

For finite size metal structures, surface plasmon polaritons can couple to free space waves due to the abrupt geometric changes at the edges which can provide momentum match between evanescent waves and free space waves. We simulated the electromagnetic field distribution of the structure shown in Figure S15a, where an electric dipole placed at the center of graphene to represent the center bright spot. In our simulation, the size of gold electrodes and $\mathrm{hBN}$ are $50 \mathrm{~nm}$ (height) $\times 3 \mu \mathrm{m}$ (width) and $20 \mathrm{~nm}$ (height) $\times 10 \mu \mathrm{m}$ (width), respectively. Drude dispersion model is used to describe the relative permittivity of gold, with plasma frequency $1.36 \times 10^{16} \mathrm{rad} / \mathrm{s}$ and damping frequency $2.73 \times 10^{13} \mathrm{rad} / \mathrm{s}$. The frequency of the electric dipole is fixed as $4.28 \times 10^{14} \mathrm{~Hz}$ $(700 \mathrm{~nm})$. Figure $\mathrm{S} 15 \mathrm{~b}$ shows the intensity distribution of electric filed in y direction, which clearly shows that the surface plasmon polaritons modes at the surface of gold electrodes is excited by the free space waves originated from the electric dipole. This indicates that the surface plasmon polaritons modes at the surface of gold electrodes can couple to free space waves. For comparison, without the gold electrodes, the intensity distribution of electric filed in $y$ direction is shown in Figure S15c. 

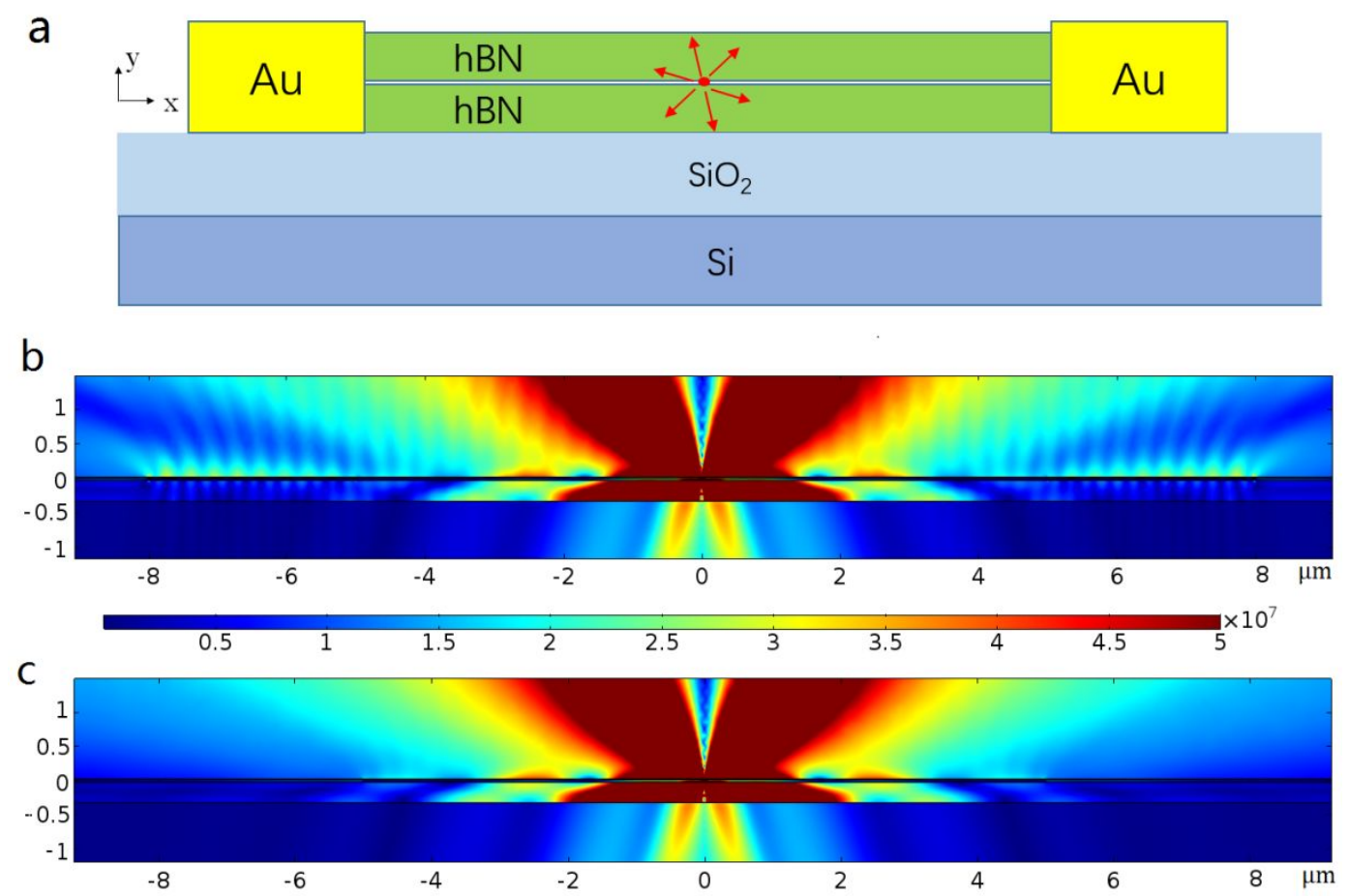

Figure S15. Simulation of the electromagnetic field distribution in $\mathrm{hBN} /$ graphene $/ \mathrm{hBN}$ thermal emitter a Schematic diagram of the device structure for simulation. b Intensity distribution of electric filed in y direction for the structure shown in a. c Intensity distribution of electric filed in $y$ direction without the gold electrodes.

\section{Section 5. Enhanced emission by a photonic cavity}

The spectra of thermal emission of graphene can be approximated by Planck's law, modified by emissivity $\epsilon\left(\lambda, T_{e}\right)$.

$$
I_{\text {measured }}\left(\lambda, T_{e}\right)=\epsilon\left(\lambda, T_{e}\right) I_{b}\left(\lambda, T_{e}\right)
$$

where $\left.I_{b}\left(\lambda, T_{e}\right)=8 \pi h c / \lambda^{5} \cdot{ }_{1}{ }_{\left(e^{h c} / \lambda k_{B} T_{e}\right.}-1\right)$ is the black-boby raditon spectra. The dielectric layers of graphene encapsulated by $\mathrm{hBN}$ or covered by $\mathrm{Al}_{2} \mathrm{O}_{3}$ on $\mathrm{SiO}_{2}$ and $\mathrm{Si}$ serve as a photonic cavity which modify the thermal emissivity $\epsilon\left(\lambda, T_{e}\right)$ and then shape the spectra of thermal radition. 
According to the Kirchoff's law, the thermal emissivity $\epsilon\left(\lambda, T_{e}\right)=\alpha\left(\lambda, T_{e}\right)$, where $\alpha\left(\lambda, T_{e}\right)$ is the absorptiviy of graphene. Considering that the thickness of graphene and $\mathrm{hBN}$ is much smaller than the wavelength, the consitriction has negligible influence on the mode field distribution of the photonic cavity. For simplicity, we ignore the graphene consitriction and use a multilayer slab model to calculate the absorptivity. In our calculation, the thickness of two layers of $\mathrm{hBN}, \mathrm{SiO}_{2}$ is $25 \mathrm{~nm}, 25 \mathrm{~nm}$ and $300 \mathrm{~nm}$, respectively. The refractive indices of $\mathrm{hBN}, \mathrm{SiO}_{2}$ and $\mathrm{Si}$ were taken to be 2.2, 1.46 and 3.5 respectively. We use Kubo formula to describe the conductivity of graphene, which is temperature dependent. For a detector subtending angle $\theta_{\max }$, the total absorptivity is

$$
\alpha\left(\lambda, T_{e}\right)=2 \pi \Sigma_{T E, T M} \int_{0}^{\theta_{\max }} \alpha\left(\lambda, \theta, T_{e}\right) \sin \theta \cos \theta d \theta
$$

We use finite-element method-based software (COMSOL Multiphysic) to calculate the absorptivity. Then we fit the measured thermal radiation spectra to with only the elctrons temperature as the fitting parameter.

\section{Section 6. Resonant emission at telecom spectrum range}

According to Kirchhorr's law of thermal radiation, for an arbitrary body emitting and absorbing thermal radiation in thermodynamic equilibrium, the emissivity is equal to the absorptivity. Because the absorption of monolayer graphene toward the incident light has significantly increased from $2.3 \%$ to $100 \%$ by coupling graphene with some resonant structures, these resonant absorption structures could also be utilized to improve the emissivity of monolayer graphene when graphene has been heated to high temperature.

Here, we present a kind of resonant emission structure which could change the peak emission wavelength of graphene around $1550 \mathrm{~nm}$. The schematic image of the emission structure is 
demonstrated in Figure S16a. The structure comprised monolayer graphene which is protected by two hBN layers and a silica layer that was etched with patterned holes, and a gold layer is coated in the back side of the silica layer. The proposed graphene-based resonant emission structure is analyzed by using finite-element method-based software (COMSOL Multiphysic). In the simulation, the monolayer graphene was regarded as a conductive surface with optical conductance of $\mathrm{G}_{0} \approx 6.08 \times 10^{-5} \Omega^{-1}$. The refractive indices of $\mathrm{hBN}$ and silica were taken to be 2.2 and 1.45 , and the dielectric constant of gold was given by the Drude model as $\varepsilon(\omega)=\varepsilon_{\infty}-\omega_{\mathrm{p}}^{2} /\left(\omega^{2}+i \gamma \omega\right)$ with $\varepsilon_{\infty}$ $=1.0, \omega_{\mathrm{p}}=1.37 \times 10^{16} \mathrm{~s}^{-1}$ and $\gamma=8.17 \times 10^{13} \mathrm{~s}^{-1}$. The thicknesses of $\mathrm{hBN}$, silica and gold layers are taken as $20 \mathrm{~nm}, 510 \mathrm{~nm}$ and $200 \mathrm{~nm}$, respectively. The radius, depth and period of the hole are chosen to be $340 \mathrm{~nm}, 400 \mathrm{~nm}$, and $1350 \mathrm{~nm}$, respectively. Figure S16b shows the spectrum of total absorption and graphene in the proposed structure.

The final graphene radiation spectrum is obtained by multiplying the blackbody emission spectrum with the emissivity (absorption) of monolayer graphene in the proposed resonant structure. As shown in Figure. S17a-d, red solid line and black dashed line represent the radiation existence $\left(\mathrm{M}_{\mathrm{eb}}(\lambda, \mathrm{T})\right)$ of monolayer graphene with and without the resonant structure. It is clear that the $\mathrm{M}_{\mathrm{eb}}(\lambda, \mathrm{T})$ around $1550 \mathrm{~nm}$ of graphene in the resonant emission structure is 40 times bigger than that without resonant emission structure at any temperature, which verifies that resonant structures are an efficient method to control the emission wavelength and intensity of monolayer graphene. 

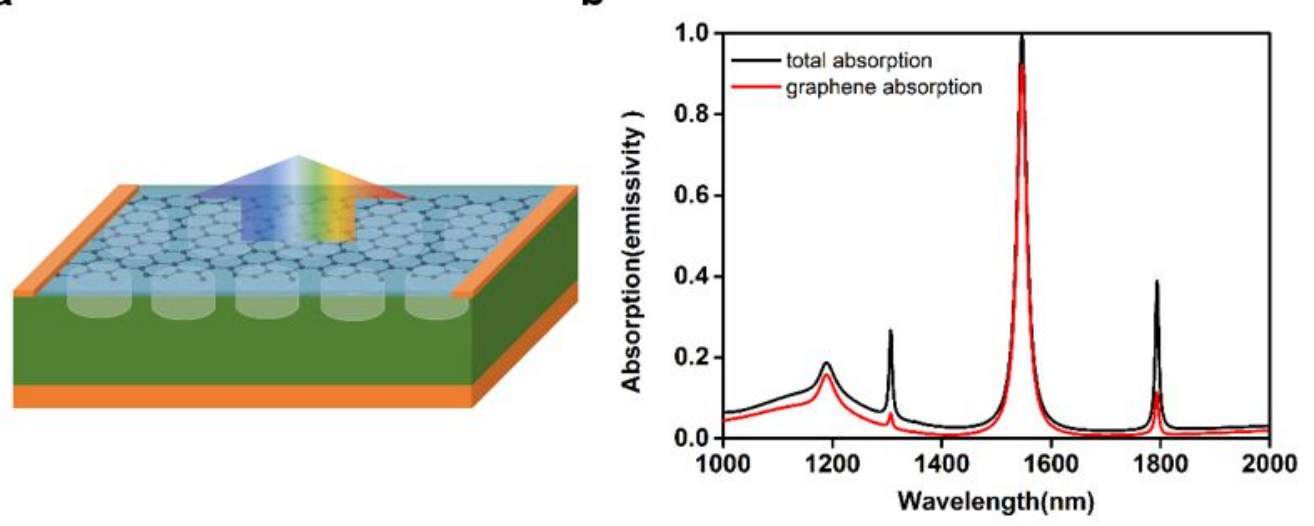

Figure S16. a Schematic of the graphene-based resonant emission structure. b The absorption ( emissivity) of the total absorption and graphene.

a

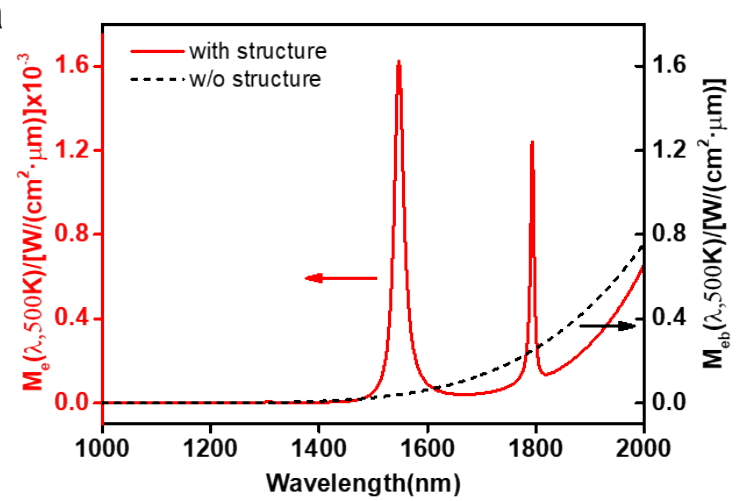

b

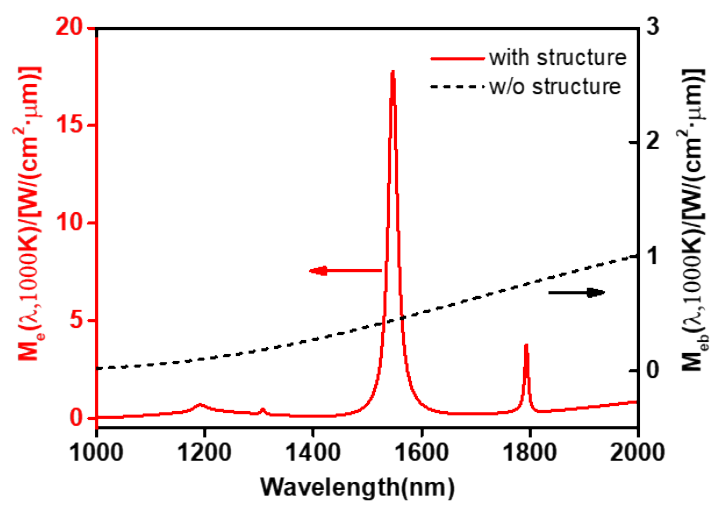

d

C

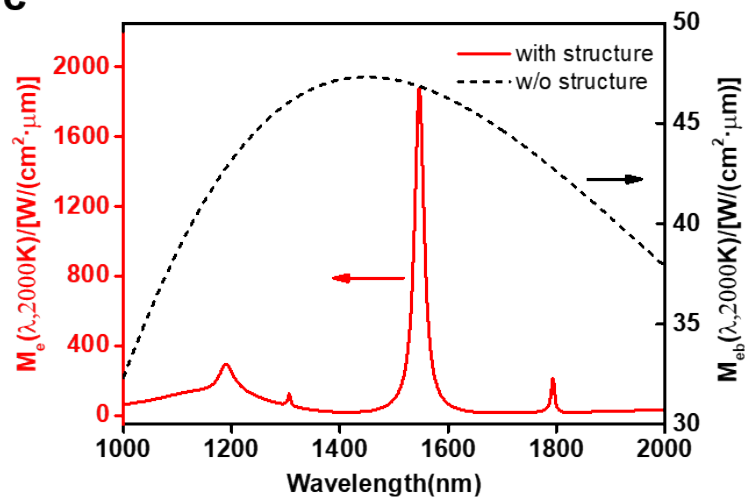

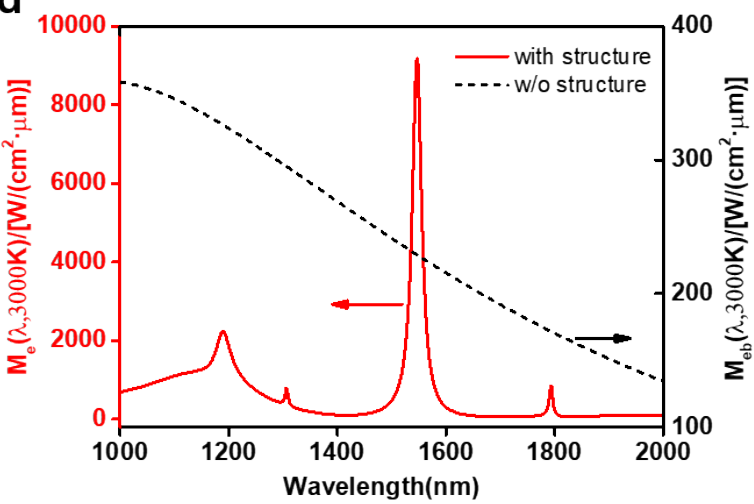

Figure S17. The emission spectra of graphene with and without the resonant structure at temperature of a $500 \mathrm{~K}$, b $1000 \mathrm{~K}$, c $2000 \mathrm{~K}$, d $3000 \mathrm{~K}$, respectively. 